Document downloaded from:

http://hdl.handle.net/10251/56940

This paper must be cited as:

Catalá Civera, JM.; Canós Marín, AJ.; Plaza González, PJ.; Gutiérrez Cano, JD.; García Baños, B.; Penaranda-Foix, FL. (2015). Dynamic Measurement of Dielectric Properties of Materials at High Temperature During Microwave Heating in a Dual Mode Cylindrical Cavity. IEEE Transactions on Microwave Theory and Techniques. 63(9):2905-2914. doi:10.1109/TMTT.2015.2453263.

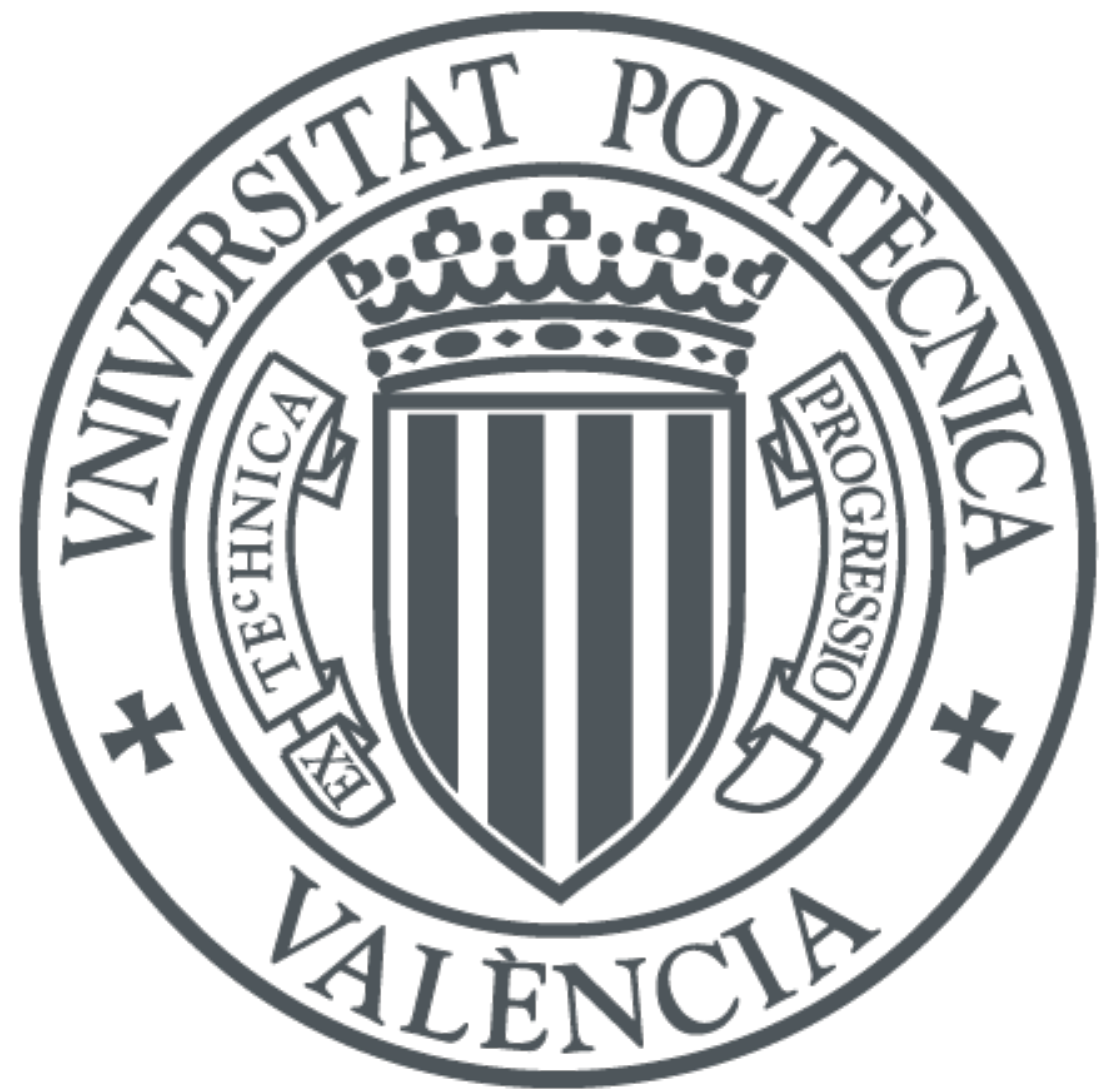

The final publication is available at

http://dx.doi.org/10.1109/TMTT.2015.2453263

Copyright Institute of Electrical and Electronics Engineers (IEEE)

Additional Information 


\title{
Dynamic Measurement of Dielectric Properties of Materials at High Temperature During Microwave Heating in a Dual Mode Cylindrical Cavity
}

\author{
José M. Catalá-Civera, Senior Member, IEEE, Antoni J. Canós, Pedro Plaza-González, \\ José D. Gutiérrez, Beatriz García-Baños, and Felipe L. Peñaranda-Foix, Senior Member, IEEE
}

\begin{abstract}
A microwave cavity and heating system for microwave processing and in situ dynamic measurements of the complex permittivity of dielectric materials at high temperatures $\left(\sim 1000{ }^{\circ} \mathrm{C}\right)$ has been developed. The method is based on a dual-mode cylindrical cavity where heating and testing are performed by two different swept frequency microwave sources. A cross-coupling filter isolates the signals coming from both sources. By adjusting the frequency bandwidth of the heating source and the level of coupling to the cavity, an automatic procedure allows for the establishment of a desirable level of heating rate to the dielectric sample to reach high temperatures in short cycles. Dielectric properties of materials as a function of temperature are calculated by an improved cavity perturbation method during heating. Accuracy of complex permittivity results has been evaluated and an error lower than $5 \%$ with respect to a rigorous analysis of the cavity has been achieved. The functionality of the microwave dielectric measurement system has been demonstrated by heating and measuring glass and ceramic samples up to $1000{ }^{\circ} \mathrm{C}$. The correlation of the complex permittivity with the heating rate, temperature, absorbed power, and other processing parameters can help to better understand the interactions that take place during microwave heating of materials at high temperatures compared to conventional heating.
\end{abstract}

Index Terms-Cavity pertubation method, dielectric properties, high temperature, microwave heating.

\section{INTRODUCTION}

$\mathbf{M}$ EASUREMENT of dielectric properties of materials at high temperatures is central for numerous industrial, scientific, and medical (ISM) applications, including microwave communications.

The design of microwave processing systems, in particular, requires knowledge of the dielectric properties of materials since they influence the ability of the material to absorb microwave energy and transfer it into heat. However, these

Manuscript received February 17, 2015; revised May 08, 2015 and June 22, 2015; accepted June 27, 2015. This work was supported by the Ministerio de Economía y Competitividad (MINECO) under Project TEC2012-37532-C02-01 and by the European Regional Development Funds (ERDF).

The authors are with the Institute for the Applications of Advanced Information and Communication Technologies (ITACA), Universitat Politècnica de València, 46022 València, Spain (e-mail jmcatala@dcom.upv.es; ajcanos@dcom.upv.es; pedplago@itaca.upv.es; jdgutierrez@itaca.upv.es; beagarba@upvnet.upv.es; fpenaran@dcom.upv.es).

Color versions of one or more of the figures in this paper are available online at http://ieeexplore.ieee.org.

Digital Object Identifier 10.1109/TMTT.2015.2453263 properties are dependent on the temperature and they change when the material is heated [1]-[3].

Although microwave heating has been used for many decades, the mechanisms of microwave and matter interactions are still incompletely understood, especially at high temperatures. One reason might be the lack of specific and advanced instrumentation designed for this purpose [4].

To overcome the practical limitations imposed by the high temperatures of samples, the methodologies normally applied for dielectric measurements at low temperature require some modification. These methodologies are very influenced by the heating technique employed to provide the high temperatures to the samples. Some of the reported techniques include: conventional oven heating, infrared (IR) heating, laser heating, and also microwave heating [5].

For example, a conventional furnace placed on the top of a (TM0n0) cylindrical cavity is used for heating small cylindrical samples inserted in silica holders [6]. Once the desired temperature is reached in the furnace, the holder is moved to the cavity for dielectric measurements and then rapidly moved back to the furnace. Since some cooling of the sample occurs during transfer from the furnace to the cavity, the real temperature measurement is estimated from cooling curves.

The cavity perturbation method (CPM) is reported to measure the complex permittivity from the resonance measurements. Accordingly, the CPM has become one of the most popular methods for calculating the dielectric properties under these temperature conditions [5].

The use of microwave for both heating and testing allows short and fast heating cycles with lower thermal gradients and at the same time in-situ dynamic sample measurements during sample heating [5].

In previous works, Couderc et al. developed a dual-mode cylindrical cavity technique using a heating mode of $\mathrm{TM}_{010}$ at $2.45 \mathrm{GHz}$ and a measurement mode of $\mathrm{TE}_{111}$ at $3.1 \mathrm{GHz}$ for spherical samples, and $\mathrm{TM}_{012}$ at $3.7 \mathrm{GHz}$ for rod samples [7]. The heating and diagnostic modes were individually coupled to the cavity with minimum cross-coupling.

$\mathrm{Xi}$ and Tinga reported a dual-mode coaxial quasi TEM re-entrant cavity as a microwave heater and as a microwave dielectrometer for high-temperature measurements of small samples [8]. In this experimental design, the diagnosis mode employed a swept frequency source and a magnitude detector for the measurement of the cavity resonance and dielectric determinations 
by the CPM, and a single continuous wave (CW) frequency power source for the heating mode. In both cases, complicated and inefficient electronic tuners were required during heating to adjust the power source $(\mathrm{CW})$ frequency to the resonant frequency of the cavity due to temperature-dependent changes in the sample properties.

To reduce the complexity of the dual process, Jow et al. developed a $\mathrm{TM}_{012}$ single mode cavity for microwave processing and measurement of the properties of polymers at low temperatures with a swept frequency microwave power source (2.4-2.6 $\mathrm{GHz}$ [ [9]. Dielectric properties were determined simultaneously by the CPM from the resonance power absorption curve formed by the measured reflected signals in the cavity through a directional coupler.

Nesbitt et al. reported a cylindrical cavity operating in the $\mathrm{TE}_{111}$ single mode around the resonant frequency of $2.45 \mathrm{GHz}$ for in situ diagnosis of cure reactions during microwave heating [10]. A narrowband (2.3-2.7 GHz) solid-state amplifier fed the cavity and the transmission signal in the cavity was monitored through a coupling loop with a network analyzer, which allowed the measurement of cavity resonance during heating for simultaneous determination of dielectric properties by the CPM.

The same principle was used by Guan and Nikawa with a $\mathrm{TM}_{010}$ single mode cylindrical cavity around the frequency of $2.45 \mathrm{GHz}$ for heating and measuring the dielectric properties of some ceramics at high temperatures [11]. In this setup, the signal from the first port of the network analyzer was amplified with a power source for heating, and by means of a coupling loop, the transmission response was measured with the second port of the network analyzer for dielectric calculations by the CPM. The use of a single source for simultaneous heating and measuring operations reduces the complexity of the dual operation, but complicates the measurement of the cavity resonance. This applies mainly for high-loss materials, where the resonance response might disappear completely.

In this paper, a fast method for determining the dielectric properties of materials at high temperature $\left(\sim 1000{ }^{\circ} \mathrm{C}\right)$ in real time is described. The method consists of a dual-mode microwave cavity, where heating and measuring is performed simultaneously with two different microwave sources. The conflict between heating and testing procedures has been avoided by the design of a cross-coupling filter that provides a high isolation between both cavity modes. To ensure an efficient power delivery to the microwave cavity during processing of a dielectric sample at high temperatures, an automatic procedure adjusts the sweep frequency band of the input power source and the degree of coupling to the cavity contingent on the desired sample heating rate. The heating system provides faster and more robust measurements in a wider temperature and materials range of application than previous dual-mode systems.

Dielectric properties are calculated by an enhanced CPM, which takes into account the depolarization of the electric field into the sample by a quasi-static (QS) approximation [12], and a new formula is suggested to remove the effect of the wall losses of the testing cavity. The accuracy of dielectric measurements has been evaluated by comparing the results given by the CPM with a rigorous solution of the cylindrical cavity [13].

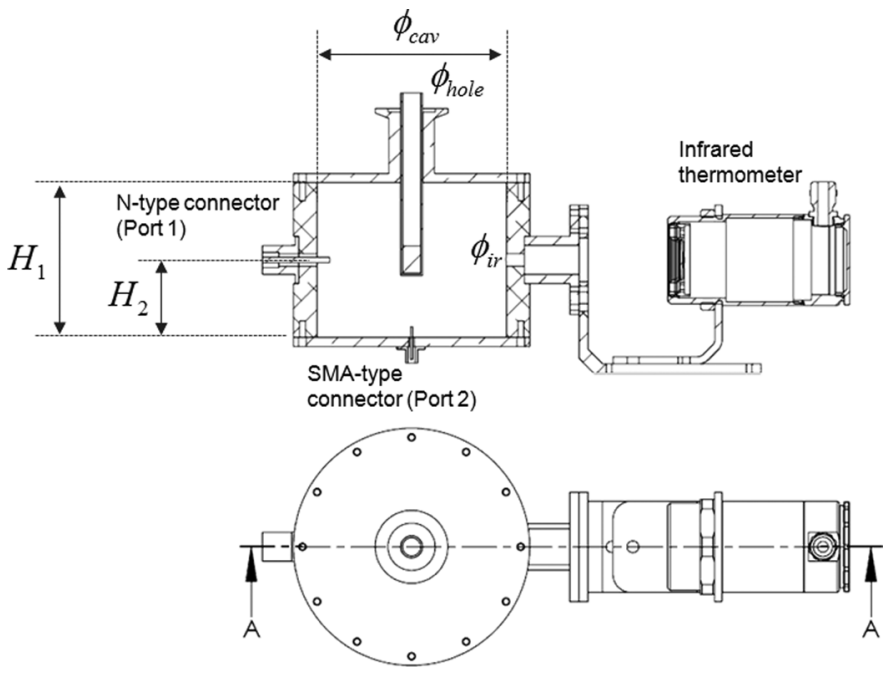

Fig. 1. Schematic view of the dual-mode cylindrical cavity. $H_{1}=85 \mathrm{~mm}$ $H_{2}=H_{1} / 2, \phi_{\text {cav }}=104.92 \mathrm{~mm}, \phi_{\text {hole }}=12.1 \mathrm{~mm}$, and $\phi_{i r}=7 \mathrm{~mm}$.

Experimental results of complex permittivity of ceramic and glass samples are given in Section $\mathrm{V}$ for temperatures varying from room temperature to $1000{ }^{\circ} \mathrm{C}$.

\section{Microwave CAVITy Design}

The microwave cavity test cell has been designed to have two dominant modes. In this dual configuration, one of the resonant modes is used for heating a sample of dielectric material with a high power signal and the second resonant mode measures the dielectric properties with low power signals. Unlike previous dual designs [7], the microwave cavity has been designed to heat in the cylindrical $\mathrm{TE}_{111}$ mode and to measure in the $\mathrm{TM}_{010}$ mode. Both cylindrical modes could be used for heating or measuring separately because both resonant modes exhibit a strong and uniform electric field where the test sample is positioned. However, $\mathrm{TM}_{010}$ is selected here as the measuring mode because it causes a larger frequency shift as a function of the sample dielectric properties, and therefore, higher sensitivity in the dielectric measurements can be accomplished.

Fig. 1 shows the schematic view of the dual-mode cylindrical microwave cavity designed for high-temperature dielectric heating and permittivity measurements.

The dimensions of the cavity must be designed carefully to avoid other disturbing modes. The heating mode was selected to have a resonance around the ISM frequency of $2.45 \mathrm{GHz}$ for future industrial application of the measurement results. Cavity diameter and height were fixed to 104.92 and $85 \mathrm{~mm}$, respectively. The theoretical resonant frequencies of both modes were calculated by analytical expressions [14], resulting in a value of $2.432 \mathrm{GHz}$ for the $\mathrm{TE}_{111}$ mode and $2.187 \mathrm{GHz}$ for $\mathrm{TM}_{010}$ mode.

Fig. 2 shows the normalized analytical electric field distribution of both resonant modes in the empty cavity. The $E$-field vector of the $\mathrm{TE}_{111}$ mode is perpendicular to the cavity axis with maximum field magnitude at the center of the cavity. The $E$-field vector of the $\mathrm{TM}_{010}$ mode is parallel to the cavity axis, also with maximum magnitude at the cavity center. The figure 


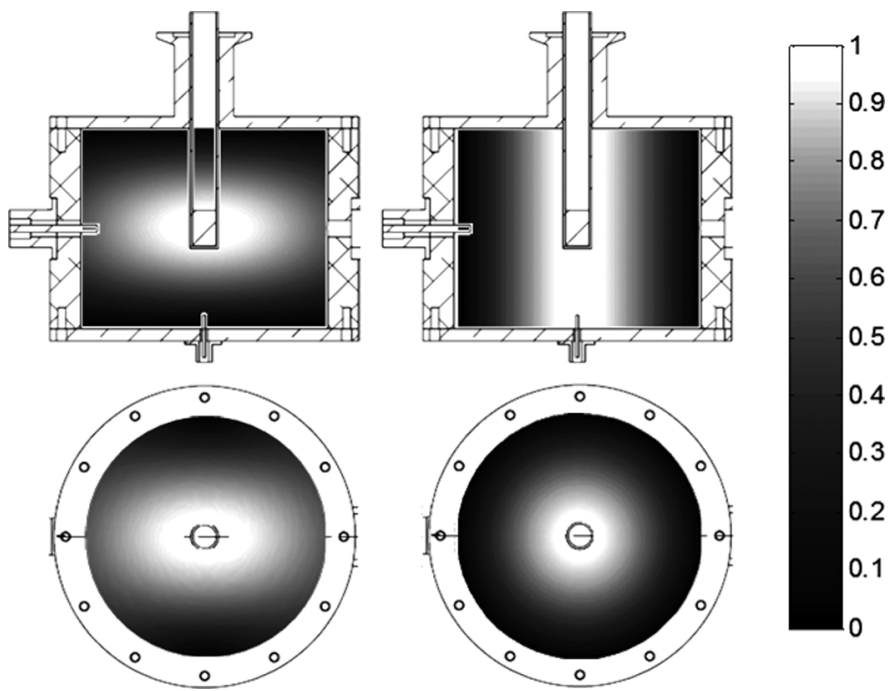

Fig. 2. Normalized analytical $E$-field magnitude in the dual-mode cylindrical microwave cavity. $\mathrm{TE}_{111}$ mode (left) and $\mathrm{TM}_{010}$ mode (right).

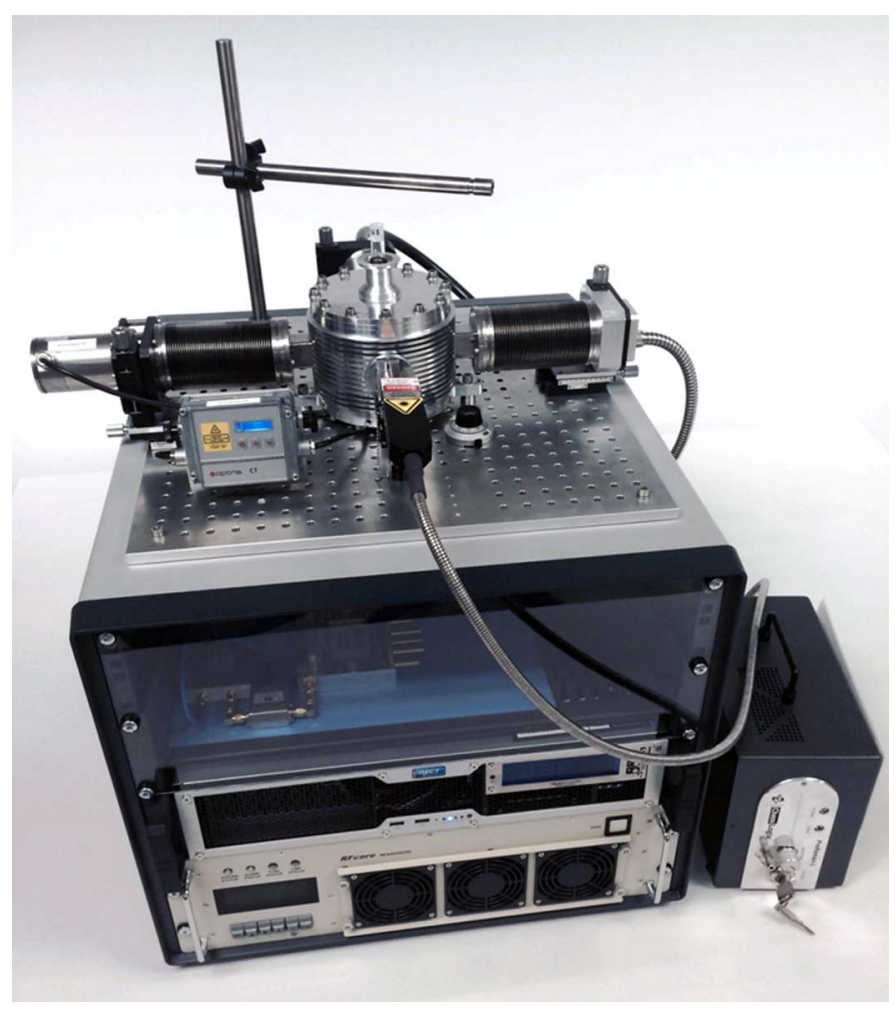

Fig. 3. Photograph of the manufactured dual mode microwave cavity.

also displays the location of the sample in relation to the maximum of $E$-field for each mode to ensure uniform processing and testing. The sample size was fixed around $10 \mathrm{~mm}$ in diameter and $15 \mathrm{~mm}$ in height, in accordance with the $E$-field representation.

The sample of material is held in a quartz vial (inner diameter $\sim 10 \mathrm{~mm}$, external diameter $\sim 12 \mathrm{~mm}$ ), capable of handling high temperatures $\left(\sim 1300{ }^{\circ} \mathrm{C}\right)$, which is inserted within the cavity through a cutoff hole located at the central plane of the top wall. An IR thermometer is used to measure the surface temperature of the dielectric sample from

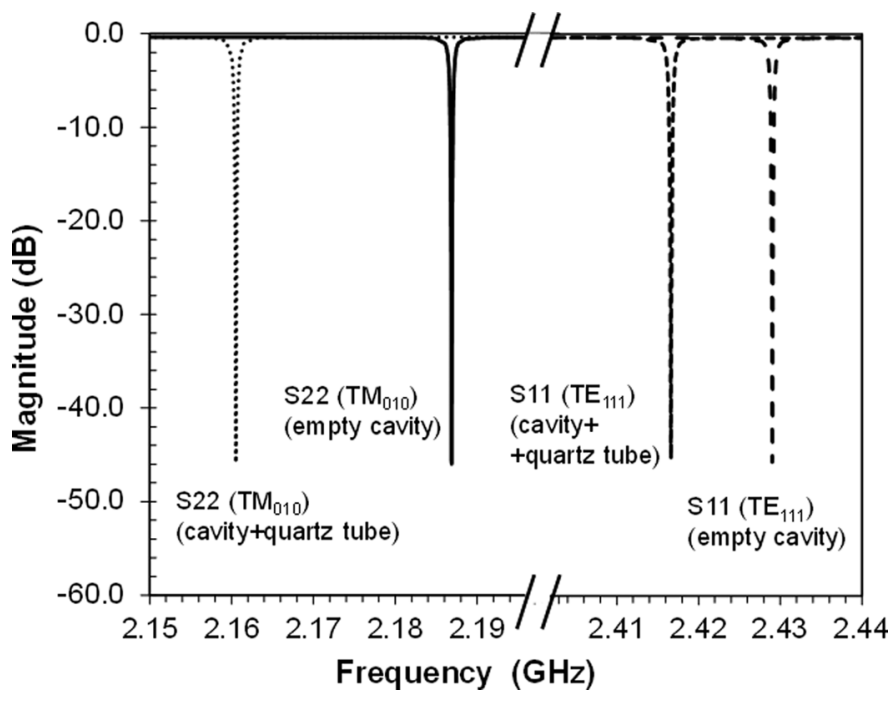

Fig. 4. Measured magnitude of the scattering parameters from heating Port 1 $\left(S_{11}\right)$ and measurement Port $2\left(S_{22}\right)$ of the dual-mode resonant cavity (empty and with a quartz vial).

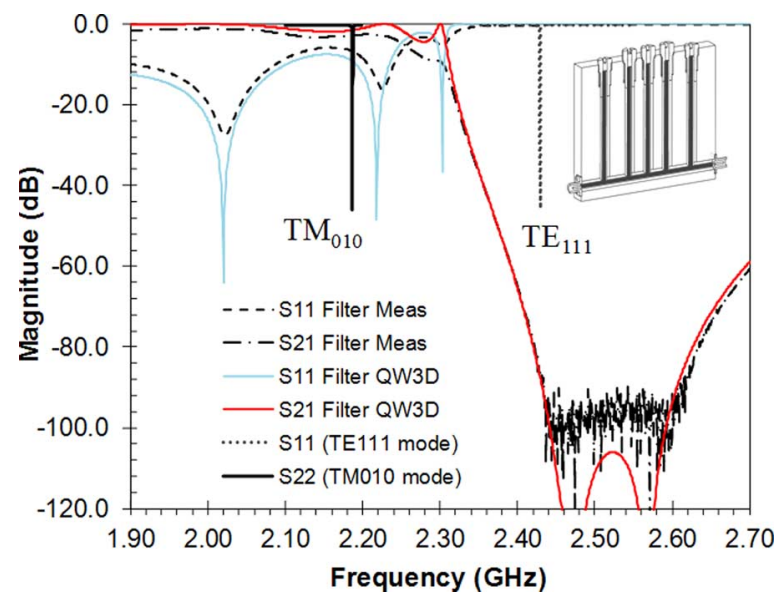

Fig. 5. Magnitude of scattering parameters of the cross-coupling filter with the resonant peaks of the heating and measurement modes in the cavity.

outside of the cavity through a 7-mm-diameter cutoff hole positioned in the lateral wall of the cavity. A third 7-mm hole is used to place a video camera to observe the dielectric sample during processing. The dimensions and positions of access holes in the cavity were designed to ensure that they do not disturb the fields or resonances of both modes, and to prevent microwave leakage.

To avoid cross-coupling between heating and testing operations in the dual mode cavity, both resonant modes must be well separated in frequency, and the type and position of suitable coupling devices of both modes must be designed for maximum isolation between them.

The high power signal ( $\mathrm{TE}_{111}$ mode) is fed into the cavity via an electric probe placed at the sidewall through an $N$-connector (inner diameter $\sim 3 \mathrm{~mm}$ ). The low-level measurement signal ( $\mathrm{TM}_{010}$ mode) is coupled to the cavity by a second electric probe located at the central plane of the bottom wall using an SMA connector (inner diameter $\sim 1.5 \mathrm{~mm}$ ). The cross-sectional diagram of the cavity is shown in Fig. 1 and indicates the 


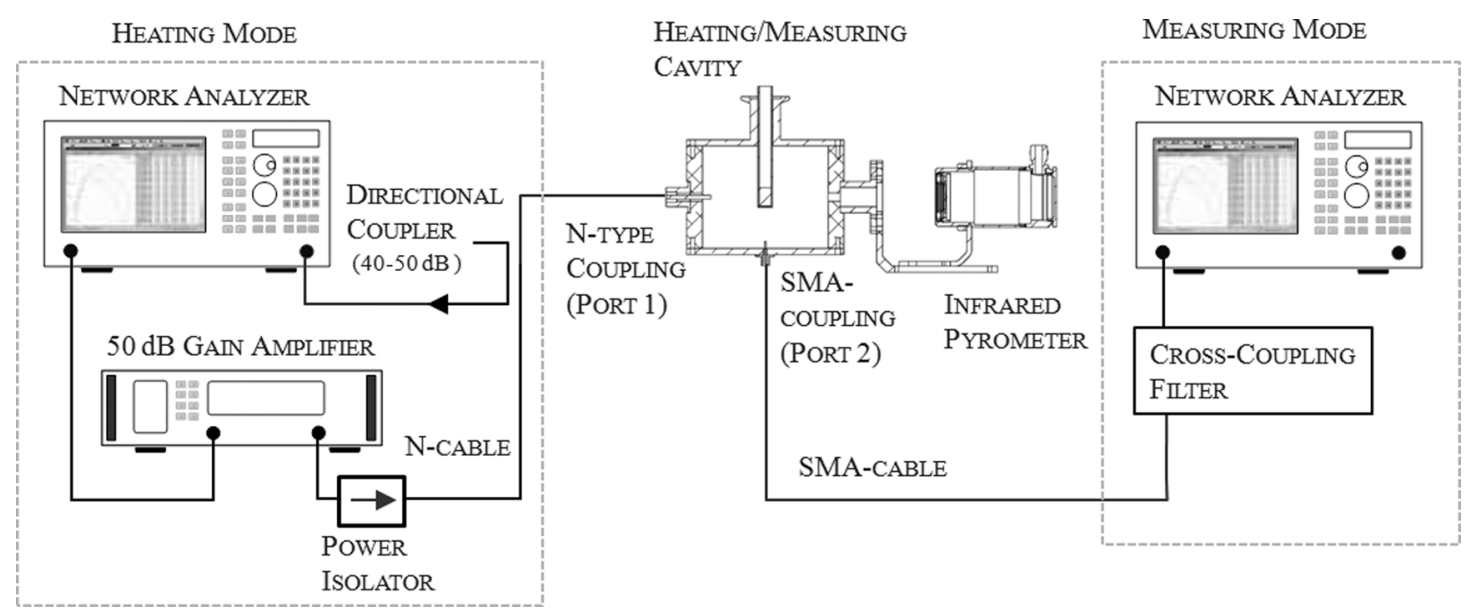

Fig. 6. Schematic of the experimental setup used for heating and dielectric measurements in a dual-mode microwave cavity.

relative positions of the electric coupling probes ( $N$-type connector, Port 1; SMA-type connector, Port 2). Both probes are mounted on a mobile mechanical device (not displayed in Fig. 1) to modify penetration into the cavity, allowing adjustment to the desirable level of coupling. Fig. 3 shows a photograph of the manufactured cavity with the mobile coupling devices and a Raman spectrometer.

Fig. 4 shows a measurement of scattering parameters of both ports in the cavity by adjusting the penetration of probes close to critical coupling. Both resonant frequencies are displayed around the theoretical value with a small drift caused by the equivalent circuit of the coupling network [15] and the access holes. The figure also illustrates the frequency shift of each mode when an empty quartz vial is inserted into the cavity. The perpendicular (cross-polarized) configuration between both coupling probes, related to the electromagnetic (EM) field patterns, causes an approximate isolation $\left(S_{21}\right)$ between modes of $30-45 \mathrm{~dB}$, measured with different penetrations of the probes in the cavity. Therefore, an additional low-pass filter must be designed to further attenuate the heating mode in order to ensure an estimated isolation of $100 \mathrm{~dB}$, required to avoid interference into the measurement channel.

\section{A. Cross-Coupling Filter}

Since the frequency of the heating mode $(\sim 2.432 \mathrm{GHz})$ and the frequency of the testing mode $(\sim 2.187 \mathrm{GHz})$ are very similar, the cross-coupling filter requires a rejection level greater than $60 \mathrm{~dB}$ on the side of the heating band, and at the same time, very low insertion losses around the testing band (lower than $3 \mathrm{~dB}$ ). The proposed cross-coupling filter is a coaxial stub filter with five sections. In this configuration, five short-circuited coax stubs are directly folded to the main coaxial line as parallel shunts, (shown in the inset of Fig. 5). The $N$ connector-type coaxial structure has been chosen because it is capable of handling the high power required for a safe operation.

The structure has been modeled using the finite-difference time-domain (FDTD) QuickWave-3D (QW3D) EM commercial simulator and the position and length of stubs were optimized according to the specifications described above.
Fig. 5 shows the QW3D modeling results of scattering parameters of the proposed filter (solid line), where a rejection level of $80 \mathrm{~dB}$ for the frequency of the heating mode is achieved. The same figure shows the results obtained from the measurements of the structure (dashed line) and the measurement of the two cavity resonant modes. Both responses (modeled and measured) are in good agreement, confirming the validity of the suggested design.

\section{EXPERIMENTAL SETUP}

The high isolation achieved with the filter between both modes of the microwave cavity allows sample heating and measurement of dielectric properties to be performed simultaneously with two separated microwave sources by the method described below. Fig. 6 shows the block diagram of the heating/measurement system

The microwave heating source is a narrowband (2.2-2.6 GHz) solid-state amplifier (RCA2026U50, RFcore Ltd.) driven by the RF output of a vector network analyzer (VNA) (Rohde \& Schwarz ZVRE). The network analyzer output delivers an output of $0 \mathrm{dBm}(1 \mathrm{~mW})$ and the gain of the amplifier is around $50 \mathrm{~dB}$. The maximum power delivered to the cavity in this configuration is $\sim 150 \mathrm{~W}$. A power isolator (Valvo VFU1045A) placed at the output of the solid-state amplifier protects the amplifier and network analyzer against power reflected from the cavity. The output of the microwave amplifier launches the microwaves into the cavity via a directional coupler and a high-power coaxial cable (SUCOFLEX 106) connected with an $N$-type connector to the coaxial probe coupling device. The directional coupler (40-dB coupling) decouples the reflected signal from the cavity to the second channel of the network analyzer, allowing the signal to be monitored. Temperature measurements of the dielectric sample through the vial container surface were done using an IR radiation thermometer (Fluent RF23) with an accuracy of $0.1{ }^{\circ} \mathrm{C}$. To prevent thermal expansion of the cavity during operation, the temperature of the cavity resonator was controlled by a water-cooling system. Fig. 3 shows a photograph of the system described (VNAs not shown). 


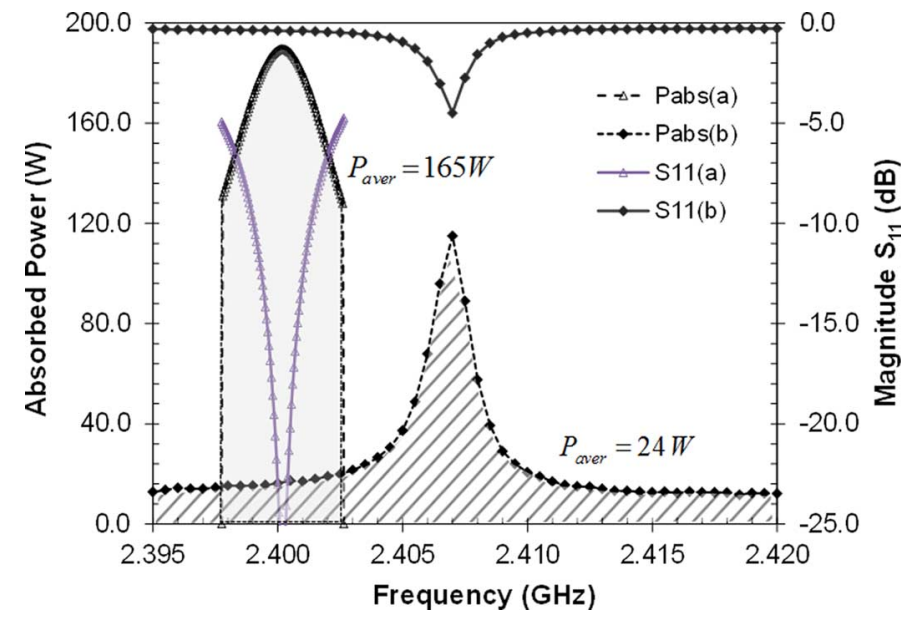

Fig. 7. Absorbed power in the heating mode of the cavity $\left(\mathrm{TE}_{111}\right)$ as a function of frequency (left) and level of coupling $S_{11}$ (right). (a) Narrow frequency sweeps of the input source. (b) Broad frequency sweeps of the input source.

The network analyzer, IR thermometer, amplifier, and variable coupling probe driver are connected to a computer via USB links such that the selection of microwave frequencies and level of coupling to feed the cavity can be adjusted either manually or automatically. Through this interconnection of the network analyzer, an open-short-line (OSL) calibration (with $N$-type high power loads) can be performed with the computer for precise determination of the input return loss in the cavity test cell system during processing.

The absorbed power in the cavity and material sample depends on the power delivered by the amplifier $P_{i}$ and the input return losses $S_{11}$ around the resonant frequency of the heating mode $\mathrm{TE}_{111}$ (Port 1 in Fig. 6).

This relation is given by the following equations:

$$
\begin{aligned}
P_{\text {abs }}\left(f_{i}\right) & =P_{i}\left(1-\left|S_{11}\left(f_{i}\right)\right|^{2}\right), \quad i=1, \ldots, N \\
P_{\text {aver }} & =\frac{1}{N} \sum_{i=1}^{N} P_{\text {abs }}\left(f_{i}\right)
\end{aligned}
$$

where $P_{\text {abs }}\left(f_{i}\right)$ is the absorbed power at a specific frequency $f_{i}$ and $P_{\text {aver }}$ is the average absorbed power for a frequency sweep in the range $\left[f_{1}<f_{i}<f_{N}\right]$ with $N$ frequency points.

Fig. 7 illustrates the absorbed power calculation in the cavity for two experimental tests with dielectric material samples, with different frequency sweeps and couplings $\left(S_{11}\right)$.

By adjusting the frequency sweeps of the VNA in a narrow band $(2.3978-2.4027 \mathrm{GHz})$ around the resonant peak of the cavity, and by adjusting the penetration of the coupling probe close to critical values $\left(S_{11}<-25 \mathrm{~dB}\right)$, almost all the output power of the amplifier is released to the cavity test cell in a manner similar to a CW source (case (a) in Fig. 7). However, for a frequency sweep covering a wider band around the resonant peak of the cavity (case (b) in Fig. 7) and a coupling of $S_{11}=-5 \mathrm{~dB}$, the absorbed power at frequencies far from the resonant peak substantially differ from those near the resonance, resulting in an effective pulsed heating.

Since the resonant frequency and the matching of the heating mode of the cavity varies as a consequence of the temperature dependence of dielectric properties of the sample, the sweeping frequencies $\left[f_{1}, f_{N}\right]$ and the probe penetration need to be adjusted continuously to track the resonant peak of the cavity during the heating cycle.

Automatic operation of this procedure has been implemented by a computer with a PID controller to provide the required absorbed power according to (1) and (2) for a desirable rate of sample heating $\left({ }^{\circ} \mathrm{C} / \mathrm{s}\right)$. Using this configuration, over 100 frequency points are feasible in less than $500 \mathrm{~ms}$.

The testing channel, used for simultaneous measurement of dielectric properties as a function of temperature, makes use of a second VNA (Hewlet Packard HP8720E) connected via the cross-coupling filter to the SMA-type coupling device of the cavity (Port 2) through an SS-402 coaxial cable (see Fig. 6). To remove the losses introduced by the filter, and systematic errors in the cables, this network analyzer is calibrated [16] in the frequency range of 1.9-2.2 GHz for measurement of the input return loss $\left(S_{22}\right)$ from which the resonant frequency and $Q$-factor of the cavity in the testing mode $\left(\mathrm{TM}_{010}\right)$ can be determined using the procedure previously described [15]. Automatic operation of these measurements is also performed by connecting the analyzer to the computer with a general-purpose interface bus (GP-IB) link. A normal programmed regime consists of 150 frequency points each $1.2 \mathrm{~s}$.

\section{High-Temperature DieleCtRic MeAsurements}

\section{A. $C P M$}

The CPM is one of the most common techniques for measuring the complex permittivity of dielectric samples at microwave frequencies [17]. This method is based on the changes in the resonance of a cavity due to the insertion of a sample of material under test (MUT). The theoretical basis of these measurements is well established, but it involves some simplifications. The main conditions for the applicability of the CPM is that the EM fields in the cavity, with and without the sample, must be approximately equal and the changes in the inverse of $Q$-factor are less than the relative shift of frequency [18].

Under this assumption and by making use of a QS approximation [12], the dielectric constant $\varepsilon^{\prime}$ and the dielectric loss factor $\varepsilon^{\prime \prime}$ of a homogeneous and isotropic material can be related to the shift in the resonant frequency $\Delta f / f$ and the change in the $Q$-factor $\Delta(1 / 2 Q)$ with the following expressions [19]:

$$
\begin{aligned}
& \varepsilon^{\prime}=1+\frac{-\frac{\Delta f}{f}\left(\eta+N \frac{\Delta f}{f}\right)-N\left[\Delta\left(\frac{1}{2 Q}\right)\right]^{2}}{\left(\eta+N \frac{\Delta f}{f}\right)^{2}+N^{2}\left[\Delta\left(\frac{1}{2 Q}\right)\right]^{2}} \\
& \varepsilon^{\prime \prime}=\frac{\eta \Delta\left(\frac{1}{2 Q}\right)}{\left(\eta+N \frac{\Delta f}{f}\right)^{2}+N^{2}\left[\Delta\left(\frac{1}{2 Q}\right)\right]^{2}}
\end{aligned}
$$

where $N \in[0.1]$ is the sample depolarization factor in the direction of the electric field polarization, which measures how much the internal field within the sample is weakened by the polarization [20]. The sample filling factor, represented by $\eta$, indicates the relative volume sample/cavity weighted with the 
squared magnitude of the $E_{0}$ field in the empty cavity resonant mode [19]

$$
\eta=\frac{\int_{v_{s}}\left|E_{0}\right|^{2} d v}{2 \int_{V}\left|E_{0}\right|^{2} d v} .
$$

The relative shift in the resonant frequency and the change in the $Q$-factor are, respectively,

$$
\begin{aligned}
\frac{\Delta f}{f} & =\frac{f_{u}-f_{u 0}}{f_{u}} \\
\Delta\left(\frac{1}{2 Q}\right) & =\frac{f_{u 0}}{f_{u}} \cdot \frac{1}{2} \cdot\left(\frac{1}{Q_{u}}-\frac{1}{Q_{u 0}}\right)
\end{aligned}
$$

where $f_{u 0}$ and $Q_{u 0}$ refer to empty cavity (unperturbed situation), while $f_{u}$ and $Q_{u}$ apply to the cavity with the specimen (perturbed situation).

Equations (3) and (4) are valid only when $E$-fields inside and outside the sample are uniform and when the skin depth and characteristic wavelength in the sample are much greater than the sample size [19].

Parameters $\eta$ and $N$ depend on the specific geometry of the cavity, material sample, and resonant mode. Although the depolarization factors of some sample geometries has been theoretically calculated in free space by a number of researchers (see, for instance, [20], [22], and [23]), they are usually not applicable with reasonable accuracy in the CPM and they need to be determined by calibration procedures; typically, by measuring materials with known permittivity [24]-[27]. The term $1 / Q_{u}-1 / Q_{u 0}$ in (7) intends to remove wall losses in the perturbed cavity by assuming that the $Q$-factor of the walls remains the same when the specimen is introduced into the cavity. Experimental results show that the insertion of small-sized and very low-loss specimens in cavities increases the $Q$-factor (due to the wall losses), thus making (7) negative, which is inconsistent with (4). An amendment of perturbation expressions was suggested in [28], where the "corrected" $Q$-factor due to losses in the cavity walls is written as

$$
Q_{u 0}^{\prime}=Q_{u 0}\left[1+\left(\varepsilon^{\prime}-1\right) \frac{v_{s}}{V}\right]
$$

where $v_{s}$ and $V$ are the volumes of the sample and cavity, respectively. However, the $Q$-factor predicted by this expression tends to be undervalued for low dielectric constants and overvalued for high dielectric constants. By considering that the increase of the $Q$-factor due to losses in walls is due to the change of stored electric energy because of the insertion of the dielectric sample, i.e., $Q_{u 0}^{\prime} \approx Q_{u 0}\left(W_{e}^{\prime} / W_{e}\right)$, where $W_{e}$ and $W_{e}^{\prime}$ are the electric energies stored in the cavity without and with a sample, respectively, and by using the resonance frequency shift predicted by the perturbation theory, the following expression is suggested here:

$$
\begin{aligned}
Q_{u 0}^{\prime} & \approx Q_{u 0}\left[1+\frac{\left(\varepsilon^{\prime}-1\right) \int_{v_{s}}|E|^{2} d v}{\int_{V}\left|E_{0}\right|^{2} d v}\right] \\
& \approx Q_{u 0}\left(2 \frac{f_{u 0}}{f_{u}}-1\right)
\end{aligned}
$$

$$
\approx Q_{u 0} \frac{f_{u 0}^{2}}{f_{u}^{2}}
$$

since $2 x-1 \approx x^{2}$ for $x \approx 1$, and $E_{0}$ and $E$ stand for the $\eta$-fields in the empty cavity and the cavity with a dielectric sample, respectively. This approach has been supported with multiple simulations of small cylindrical dielectric samples of different sizes centered in a resonant cylindrical cavity working at $\mathrm{TM}_{010}$ mode performed with commercial full-wave simulators.

Equation (7) is then rewritten as

$$
\Delta\left(\frac{1}{2 Q}\right)=\frac{f_{u 0}}{f_{u}} \cdot \frac{1}{2} \cdot\left(\frac{1}{Q_{u}}-\frac{f_{u}^{2}}{f_{u 0}^{2}} \frac{1}{Q_{u 0}}\right) .
$$

The QS perturbation analysis of samples contained in holders (such as the case of the cavity shown in Fig. 1) implies the analysis of three-phase media [23] and the precise knowledge of the permittivity and dimensions of the containers. However, the influence of sample holders in measurements is minimized by considering the cavity with the empty sample holder as the unperturbed situation [29]. Under these assumptions and by taking into account (10) for a thin and very low-loss sample holder, the relative frequency and $Q$-factor shifts to be used in (3) and (4) are expressed, respectively, as

$$
\begin{aligned}
\frac{\Delta f}{f} & =\frac{f_{u t s}-f_{u t}}{f_{u t s}} \\
\Delta\left(\frac{1}{2 Q}\right) & =\frac{f_{u t}}{f_{u t s}} \cdot \frac{1}{2} \cdot\left(\frac{1}{Q_{u t s}}-\frac{1}{Q_{u t}}-\frac{1}{Q_{u 0}} \frac{f_{u t s}^{2}-f_{u t}^{2}}{f_{u 0}^{2}}\right)
\end{aligned}
$$

where $f_{u t}$ and $Q_{u t}$ are the resonance frequency and $Q$-factor of the cavity, respectively, with the empty sample holder and $f_{u t s}$ and $Q_{u t s}$ with the sample holder containing the specimen.

The insertion hole in the cylindrical cavity shifts the resonant frequency and lowers the $Q$-factor depending on the EM fields, geometry, and material properties (near or through the hole) [30]. In the microwave cavity shown in Fig. 1, the fields in the hole remain nearly unaltered from the unperturbed to perturbed situation because the dielectric sample is far from the hole. Therefore, the effect of the insertion hole on the resonant frequency and $Q$-factor is assumed to be canceled by the perturbation expression, which involves relative deviations and $f_{u 0}$ refers, henceforth, to the resonant frequency with the insertion hole.

\section{B. Accuracy of the CPM}

The accuracy reached in dielectric determination by the CPM is difficult to evaluate. Depending on the dielectric sample, the assumptions made in the approximate theory of the perturbation of cavities are not always valid. To verify the accuracy of the dielectric calculations, a number of different samples in a wide range of dielectric constant and losses were machined in rods of 15-mm height and 9.8-mm diameter and measured inside quartz vials at room temperature by the CPM described above. The results were compared with measurements of the same samples, without the container, in a closed $\mathrm{TM}_{010}$ cylindrical cavity (diameter $=98 \mathrm{~mm}$ and height $=20 \mathrm{~mm}$ ) solved by a rigorous mode-matching method [13]. The measured complex permittivities are tabulated in Table I. 
This article has been accepted for inclusion in a future issue of this journal. Content is final as presented, with the exception of pagination.

TABLE I

Dielectric Properties of Samples $(15 \mathrm{~mm} \times 9.8 \mathrm{~mm})$ Placed Inside Quartz Vials, Calculated by the CPM and Compared With Measurements Carried Out by a Rigorous Analysis of a Closed TM T10 $_{010}$ (PARTially Filled) Cylindrical CAVity [13]

\begin{tabular}{lllllll}
\multicolumn{7}{c}{$\left(f_{u 0}=2.18762 \mathrm{GHz}, f_{u t}=2.16147 \mathrm{GHz}, Q_{u 0}=10464, Q_{u t}=10502\right)$} \\
\hline \hline Material $\left(22^{\circ} \mathrm{C}\right)$ & $f_{u t s}(\mathrm{GHz})$ & $Q_{u t s}$ & $\varepsilon_{C P M}^{\prime}$ & $\varepsilon_{C P M}^{\prime \prime}$ & $\varepsilon^{\prime} \pm \Delta \varepsilon^{\prime}$ & \multirow{2}{*}{$\varepsilon^{\prime \prime} \pm \Delta \varepsilon^{\prime \prime}$} \\
\hline \hline Ptfe & 2.15653 & 10241 & 2.066 & 0.0007 & $2.069 \pm 0.010$ & $0.00072 \pm 0.0001$ \\
\hline Rexolite & 2.15460 & 10206 & 2.549 & 0.0010 & $2.531 \pm 0.011$ & $0.00128 \pm 0.0001$ \\
\hline Nylon Fiber Glass & 2.15055 & 4121 & 3.723 & 0.0511 & $3.709 \pm 0.018$ & $0.05701 \pm 0.0011$ \\
\hline Borosilicate Glass & 2.14842 & 6392 & 4.449 & 0.0241 & $4.354 \pm 0.045$ & $0.02374 \pm 0.0006$ \\
\hline Tufnol & 2.14731 & 3666 & 4.859 & 0.0734 & $4.711 \pm 0.022$ & $0.07190 \pm 0.0009$ \\
\hline Porcelain & 2.14621 & 3875 & 5.292 & 0.0718 & $5.438 \pm 0.160$ & $0.07756 \pm 0.0037$ \\
\hline Macor & 2.14555 & 6619 & 5.569 & 0.0260 & $5.676 \pm 0.031$ & $0.02384 \pm 0.0004$ \\
\hline Alumina & 2.13930 & 10596 & 8.823 & 0.0007 & $9.007 \pm 0.051$ & $0.00025 \pm 0.0001$ \\
\hline Ptfe+Graphite & 2.12620 & 205 & 21.176 & 10.803 & $20.900 \pm 0.650$ & $9.72200 \pm 1.1300$ \\
\hline E41030-Temex Ceramics & 2.12504 & 10833 & 28.108 & 0.0006 & $29.120 \pm 0.323$ & $0.00070 \pm 0.0003$ \\
\hline E21836-Temex Ceramics & 2.12345 & 10746 & 33.258 & 0.0043 & $33.800 \pm 0.407$ & $0.00450 \pm 0.0003$ \\
\hline E5980-Temex Ceramics & 2.11851 & 10736 & 65.788 & 0.0199 & $65.806 \pm 1.266$ & $0.01834 \pm 0.0060$ \\
\hline \hline
\end{tabular}

Unloaded resonance frequencies and $Q$-factors given in Table I were determined by the procedure reported in [15]. Filling and polarization factors $(\eta=0.00238$ and $N=0.102)$ of (3) and (4) were determined by calibration with reference materials of known permittivities.

Table I also gives the combined standard uncertainties $\Delta \varepsilon^{\prime}$ and $\Delta \varepsilon^{\prime \prime}$ provided by the rigorous mode-matching method, determined according to the law of uncertainty propagation based on a Taylor expansion of first order, as stated in [31], and taking into account all the individual contributions of uncertainties in dimensions and measured magnitudes [32].

From the maximum discrepancies observed in Table I, the accuracy of the CPM is estimated to be, on average, within $3 \%$ in the dielectric constant and around $10 \%$ in the loss factor within the range $10^{-3}$ to $10^{-1}$. For very low-loss materials $\left(\Delta \varepsilon^{\prime \prime}<10^{-4}\right)$, even in cases where the $Q$-factor of the cavity with samples is higher than the value of the empty cavity, the suggested equation (12) removes the wall losses of the cavity and the discrepancies in the loss factor found for this range of losses are in the order of \pm 0.0005 .

The filling and polarization factors $(\eta, N)$ are strongly related to the dimensions of the samples employed in the calibration. For example, the application of the CPM to a sample with a deviation of $5 \%$ with respect to these dimensions (radius or height) might lead up to $40 \%$ error in the permittivity determinations.

The accuracy of permittivity calculations at high temperatures during the microwave heating cycle is estimated to be very close to the room-temperature measurements accuracy because the thermal expansion of the testing cell is negligible due to the thermal cooling and the rapid measurement cycle, and the dielectric properties of the quartz vial holder remain practically unaltered in the temperature range [33].

\section{EXPERIMENTAL RESUltS AND Discussion}

Extensive measurements have been carried out to test the performance of the method described and results for two materials are presented. The temperature dependence of the dielectric properties of glass-ceramic (Macor from Corning Inc.) and borosilicate-glass (Duran Glass from Schott AG) samples were measured from room temperature to greater than $1000{ }^{\circ} \mathrm{C}$.

Since the cavity remains cool during the heating experiments, the sample experiments temperature gradients from the center to the surface [34]. Since the IR thermometer measures the surface temperature, a calibration process based on conventional heating of samples having similar thermal properties with a thermocouple placed at the center was applied to find the relationship with the bulk temperature of the sample.

Dielectric samples were machined in rods of $15-\mathrm{mm}$ height and 9.8-mm diameter (to fit into the quartz vials) and electromagnetically processed in the heating mode of the resonant cavity. For each heating experiment, an average of 100 swept frequencies of the input source were automatically adjusted by the proportional-integral-derivation (PID) control procedure between the range of $2.33-2.43 \mathrm{GHz}$ to achieve an approximate heating rate of $0.5^{\circ} \mathrm{C} / \mathrm{s}$. The probe penetration was adjusted at the beginning of the experiment to overcouple the cavity and no additional movements were needed during the heating cycle.

Fig. 8 represents the bulk temperature reached in the Macor sample measured by the IR pyrometer. Also described is the average microwave absorbed power required by the sample and cavity for each frequency sweep, calculated according to (1) and (2) as a function of the processing time. The average microwave power absorbed by the test cell was in the range $2-90 \mathrm{~W}$ and the heating time for raising the temperature to $1100{ }^{\circ} \mathrm{C}$ was about $32 \mathrm{~min}$.

The time evolution of the resonant frequency and $Q$-factor, calculated from the measured return loss $\left(S_{22}\right)$ in the testing mode of the cavity, were used to calculate the complex permittivity of the sample for each temperature during the microwave heating cycle according to the CPM described above. Results are represented in Fig. 9. A moderate increase of dielectric constant of Macor is seen with increasing temperature and a pronounced surge occurs at around $1000{ }^{\circ} \mathrm{C}$. The dielectric loss factor follows a similar pattern and a rapid exponential increase occurs above this temperature. Specifically, this temperature $(\sim$ $1000{ }^{\circ} \mathrm{C}$ ) is related to the maximum operating temperature of the material. A comparison of the calculated results with the 


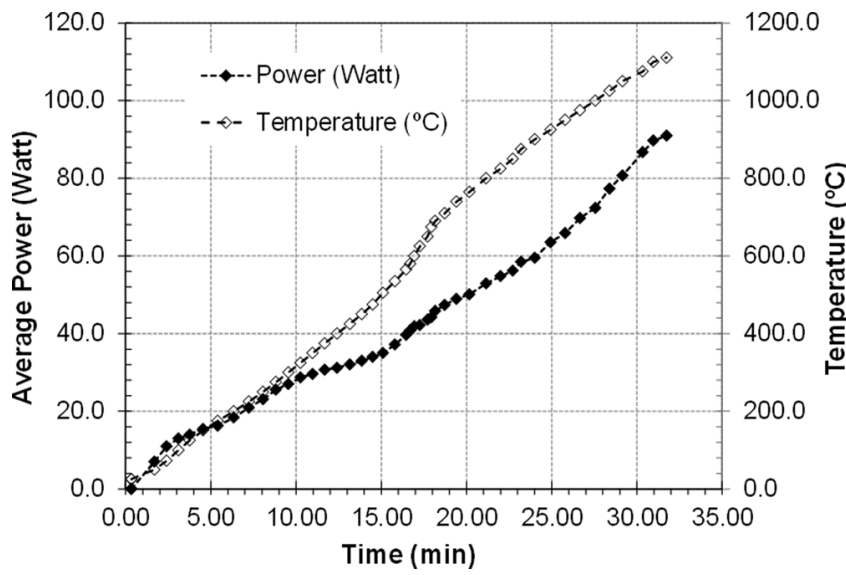

Fig. 8. Macor sample temperature and absorbed microwave power by the cavity test cell as a function of processing time.

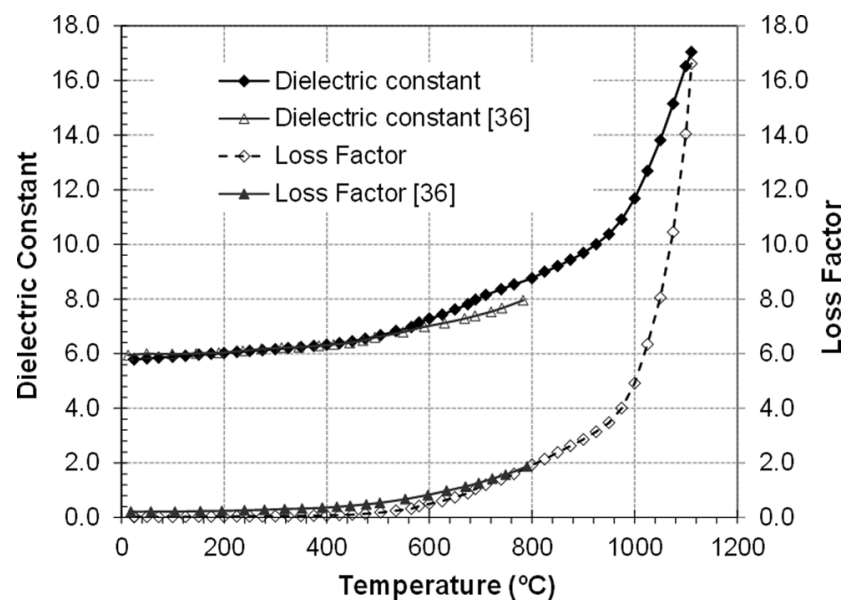

Fig. 9. Dielectric properties of Macor measured in the dual-mode cavity as a function of temperature.

experimental results of Arai et al. [35] for the same material revealed good agreement between both calculations, especially at low temperatures.

For the second material (Duran Glass), Fig. 10 represents the time evolution of the average MW absorbed power together with the sweep frequency bandwidth automatic adjustments of the input source in the heating mode of the cavity required during processing. The heating time to reach $1000{ }^{\circ} \mathrm{C}$ was around $43 \mathrm{~min}$ and the required average $\mathrm{MW}$ absorbed power was in the range of $2-75 \mathrm{~W}$.

As shown in Fig. 10, the heating process of Duran Glass requires a continuous adjustment of the source bandwidth starting from $80 \mathrm{MHz}$ at room temperature $\left(25^{\circ} \mathrm{C}\right)$ to almost $2 \mathrm{MHz}$ at $1000^{\circ} \mathrm{C}$. At these final temperatures, the high losses of the glass together with the heat losses in the cavity compel the use of a $\mathrm{CW}$ heating source, reducing the bandwidth to a small value.

Fig. 11 shows the complex permittivity of the Duran Glass sample calculated by the CPM in the testing mode of the cavity. The dielectric constant of Duran Glass, as represented in Fig. 11, exhibited a smooth monotonic increase with increasing temperature, revealing anomalies in the vicinity of the glass transition $\left(T_{g}\right)$ and melting temperatures $\left(525^{\circ} \mathrm{C}\right.$ and $860{ }^{\circ} \mathrm{C}$, respectively). Specifically, the slope of the dielectric constant in-

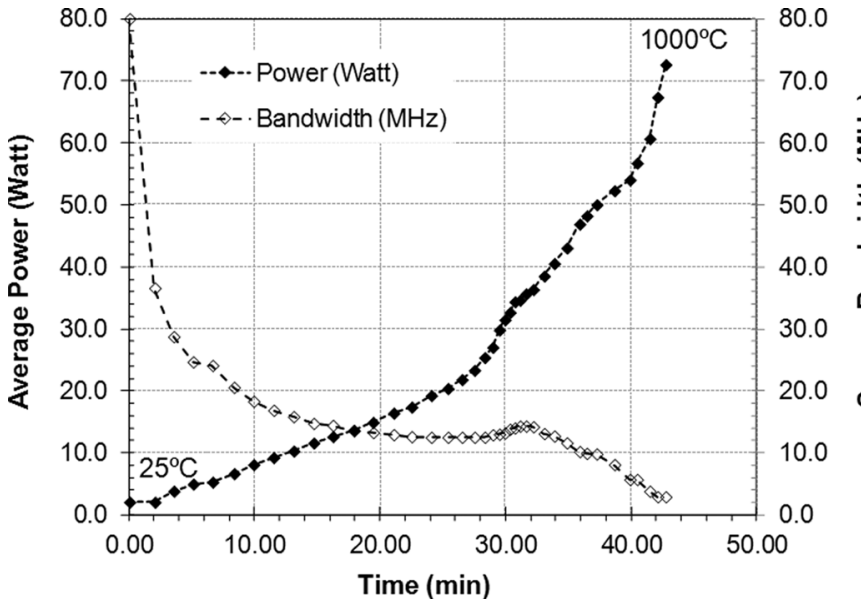

Fig. 10. Absorbed MW power and bandwidth adjustments of the input power source in the test cell as a function of processing time of Duran Glass.

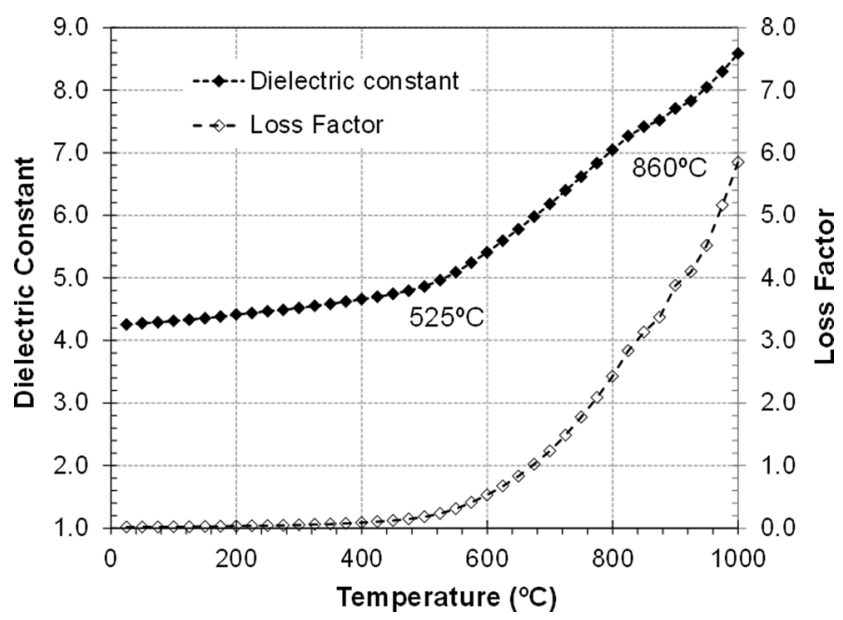

Fig. 11. Dielectric properties of Duran Glass measured as a function of temperature.

creased markedly around the transition temperature $\left(T_{g}\right)$. On the other hand, the dielectric loss factor commences with a very low value, but increases rapidly and exponentially at temperatures beyond the $T_{g}$, similar to the dielectric constant. This temperature-dependent change in dielectric properties is attributed to the transformation of the rigid supramolecular glass structure to a different structure in the viscoelastic fluid state. Both glass temperature and material melting points can be used as measurement references from specific well-documented glass compositions to verify that the calibration of the IR temperature sensor to provide bulk temperatures of the sample in the microwave cavity is correct.

In both experiments, no size changes in the dielectric samples were observed during heating; thus, the accuracy of dielectric calculations, as stated in Section IV-B, is estimated as 3\% for the real part and $10 \%$ for the imaginary part, in the entire measured range of losses.

\section{CONCLUSIONS}

A fast method for in situ and real-time measurement of the dielectric properties of materials over a wide temperature range $\left(\sim 1000{ }^{\circ} \mathrm{C}\right)$ in the microwave region has been developed. The 
method consists of a dual-mode cylindrical cavity, where the sample is heated by microwaves and measured simultaneously by two different microwave sources with no cross-coupling conflict between them.

The equipment enables a desirable level of heating rate in the sample to be fixed by adjusting, manually or automatically, the frequency bandwidth of the input source and the level of coupling to the cavity, and a precise calculation of the absorbed microwave power by the sample in the cavity is achieved. The functionality of the microwave dielectric measurement system has been demonstrated by heating and measuring glass ceramic samples up to $1000{ }^{\circ} \mathrm{C}$. Accuracies reached in the dielectric measurements by the CPM have been evaluated by comparison with more rigorous full-wave cavity procedures.

The correlation of the complex permittivity with the heating time, heating rate, temperature, absorbed MW power, and other processing parameters may help to improve the understanding of the mechanism of interactions between microwaves and materials, especially at high temperatures, and facilitates comparisons with conventionally heated materials.

\section{REFERENCES}

[1] J. G. P. Binner, T. E. Cross, N. R. Greenacre, and M. Naser-Moghadasi, "High temperature dielectric property measurements-An insight into microwave loss mechanism in engineering ceramics," in Proc. Mater. Res. Soc. Symp., 1994, pp. 247-252.

[2] S. Bringhurst, O. M. Andrade, and M. F. Iskander, "High-temperature dielectric properties measurements of ceramics," in Proc. Mater. Res. Soc. Symp., 1992, pp. 561-568.

[3] M. P. Craven, T. E. Cross, and J. G. P. Binner, "Enhanced computer modelling for high temperature microwave processing of ceramic materials," in Proc. Mater. Res. Soc. Symp., 1996, pp. 351-356.

[4] S. Vaucher, J. M. Catala-Civera, A. Sarua, J. Pomeroy, and M. Kuball, "Phase selectivity of microwave heating evidenced by Raman spectroscopy," J. Appl. Phys., vol. 99, no. 11, Jun. 2006, Art. ID 113505.

[5] L. F. Chen, C. K. Ong, C. P. Neo, V. V. Varadan, and V. K. Varadan, Microwave Electronics:Measurement and Materials Characterization. New York, NY, USA: Wiley, 2004, ch. 12, pp. 492-530.

[6] M. Arai, J. G. P. Binner, and T. E. Cross, "Comparison of techniques for measuring high-temperature microwave complex permittivity: Measurements on an Alumina/Zircona system," J. Microw. Power Elect. Energy, vol. 31, no. 1, pp. 12-18, Jan. 1996.

[7] D. Couderc, M. Giroux, and R. G. Bosisio, "Dynamic high temperatura microwave complex permittivity measurements on samples heated via microwave absorption," J. Microw. Power Elect. Energy, vol. 8, no. 1, pp. 69-82, Jul. 1973.

[8] W. G. Xi and W. R. Tinga, "Microwave heating and characterization of machinable ceramics," in Proc. Mater. Res. Soc. Symp., 1992, pp. $569-577$.

[9] J. Jow, M. C. Hawley, M. Finzel, and J. Asmussen, Jr., "Microwave processing and diagnosis of chemically reacting materials in a single-mode cavity applicator," IEEE Trans. Microw. Theory Techn., vol. MTT-35, no. 12, pp. 1435-1443, Dec. 1987.

[10] A. Nesbitt et al., "Development of a microwave calorimeter for simultaneous thermal analysis, infrared spectroscopy and dielectric measurements," Meas. Sci. Technol., vol. 15, pp. 2313-2324, Nov. 2004.

[11] Y. Guan and Y. Nikawa, "Measurement of temperature-dependent complex permittivity for materials using cylindrical resonator under microwave irradiation," Elect. Commun. Jpn., vol. 90, no. 11, pt. 2, pp. 1-8, Dec. 2007.

[12] H. M. Altschuler, Handbook of Microwave Measurements, M. Sucher and J. Fox, Eds., 3rd ed. New York, NY, USA: Polytech. Inst. Brooklyn Press, 1963, vol. 2, ch. 9.

[13] F. L. Penaranda-Foix, J. M. Catalá Civera, and A. J. Canós Marín, "Full-wave analysis of dielectric-loaded cylindrical waveguides and cavities using a new four-port ring network," IEEE Trans. Microw. Theory Techn., vol. 60, no. 9, pp. 2730-2740, Sep. 2012.

[14] C. A. Balanis, Antenna Theory Analysis and Design, 2nd ed. New York, NY, USA: Wiley, 1982.
[15] A. J. Canós, J. M. Catalá-Civera, F. L. Peñaranda-Foix, and E. de los Reyes, "A novel technique for deembedding the unloaded resonance frequency from measurements of microwave cavities," IEEE Trans. Microw. Theory Techn., vol. 54, no. 8, pp. 3407-3416, Aug. 2006.

[16] "Specifying calibration standards for the Agilent 8510 network analyzer," Agilent Technol., Santa Clara, CA, USA, Appl. Note 8510-5B, Jul. 2006.

[17] R. F. Harrington, Time-Harmonic Electromagnetic Fields. New York, NY, USA: Wiley, 2001.

[18] A. Bonincontro and C. Cametti, "On the applicability of the cavity perturbation method to high-loss dielectrics," J. Phys. E, Sci. Instrum., vol. 10, no. 2, pp. 1232-1233, Dec. 1977.

[19] S. K. Khanna, E. Ehrenfreund, A. F. Garito, and A. J. Heeger, "Microwave properties of high-purity tetrathiofulvalene-tetracyanoquinodimethan (TTF-TCNQ)," Phys. Rev. B, Condens. Matter, vol. 10, no. 6, pp. 2205-2220, Sep. 1974.

[20] L. D. Landau and E. M. Lifshitz, "Electrodynamics of continuous media," in A Course of Theoretical Physics. Oxford, U.K.: Pergamon, 1960, vol. 8 .

[21] R. Inoue, "Theoretical determination of geometrical factors in the skin depth region of enclosed cavity perturbation technique," J. Infrared, Millim., Terahertz Waves, vol. 30, no. 8, pp. 835-859, Aug. 2009.

[22] A. Sihvola, P. Ylä-Oijala, S. Järnepää, and J. Avelin, "Polarizabilities of platonic solids," IEEE Trans. Antennas Propag., vol. 52, no. 9, pp. 2226-2233, Sep. 2004.

[23] M. Beleggia, D. Vokoun, and M. De Graef, "Demagnetization factors for cylindrical shells and related shapes," J. Magn. Magn. Mater., vol. 321, no. 9, pp. 1306-1315, May 2009.

[24] F. Adams, M. de Jong, and R. Hutcheon, "Sample shape correction factors for cavity perturbation measurements," J. Microw. Power Elect. Energy, vol. 27, no. 3, pp. 131-135, Aug. 1992.

[25] V. Pohl, D. Fricke, and A. Mühlbauer, "Correction procedures for the measurement of permittivities with the cavity perturbation method," $J$. Microw. Power Elect. Energy, vol. 30, no. 1, pp. 10-26, Jan. 1995.

[26] G. Roussy, J. M. Thiebaut, F. Ename-Obiang, and E. Marchal, "Microwave broadband permittivity measurement with a multimodal helical resonator for studying catalysts," Meas. Sci. Technol., vol. 12, no. 4, pp. 542-547, Apr. 2001.

[27] M. P. Robinson, I. D. Flintoft, L. Dawson, J. Clegg, J. G. Truscott, and X. Zhu, "Application of resonant cavity perturbation to in vivo segmental hydration measurement," Meas. Sci. Technol., vol. 21, no. 1, pp. 1-10, Jan. 2010.

[28] J. Sheen, "Amendment of cavity perturbation technique for loss tangent measurement at microwave frequencies," J. Appl. Phys., vol. 102, no. 1, pp. 014102-014102-6, Jul. 2007.

[29] W. Bauhofer, "Determination of semiconductor energy gaps using the microwave cavity perturbation method," J. Phys. E, Sci. Instrum., vol. 14, no. 8, pp. 934-938, Aug. 1981.

[30] F. L. Penaranda-Foix, J. M. Catala-Civera, A. J. Canos-Marin, and B. Garcia-Banos, "Practical expression for frequency deviation due to insertion hole in cylindrical cavity," Electron. Lett., vol. 43, no. 24, pp. 1338-1340, Nov. 2007.

[31] "Evaluation of measurement data: Guide to the expression of uncertainty in measurement (GUM), " BIPM, Sèvres, France, JCGM 100:2008, 1995.

[32] F. Penaranda-Foix and J. M. Catalá-Civera, "Circuital analysis of cylindrical structures applied to the electromagnetic resolution of resonant cavities," in Passive Microwave Components and Antennas, V. Zhurbenko, Ed. Rijeka, Croatia: In-Tech, 2010, ch. 7, pp. 141-168.

[33] Y. A. Polonskii, G. A. Pavlova, V. N. Savel'ev, T. V. Milovidova, and V. B. Vinogradov, "Dielectric properties of optical quartz glass in the ultra-high frequency range and at temperatures of up to $1500{ }^{\circ} \mathrm{C}$," Glass Ceram., vol. 28, no. 3, pp. 182-184, Mar. 1971.

[34] J. Monzó-Cabrera, J. M. Catalá-Civera, A. J. Canós, and E. de los Reyes, "Simulation of temperature distributions in pressure-aided microwave rubber vulcanization processes," J. Microw. Power Elect. Energy, vol. 37, no. 2, pp. 73-88, Jul. 2002.

[35] M. Arai, J. P. Binner, G. E. Carr, and T. E. Cross, "High temperature dielectric measurements on ceramics," in Proc. Mater. Res. Soc. Symp., 1992, pp. 69-72.

José M. Catalá-Civera (M’04-SM'15) was born in Valencia, Spain, in February 1969. He received the Dipl. Ing. and Ph.D. degrees from the Universidad Politécnica de Valencia, Valencia, Spain, in 1993 and 2000, respectively.

Since 1996, he has been with the Communications Department, Universidad Politécnica de Valencia, where he received the Readership in 2000, becoming a Full Professor in 2011. He is currently the Head of the Microwave Applications 
Research Division, Institute for the Applications of Advanced Information and Communication Technologies (ITACA), Universidad Politécnica de Valencia He has coauthored about 100 papers in referred journals and conference proceedings and more than 50 engineering reports for companies. He is a reviewer of several international journals. He holds 13 patents. His research interests encompass the design and application of microwave theory and applications, the use of microwaves for electromagnetic heating, microwave resonators, measurement of dielectric and magnetic properties of materials, and development of microwave sensors for nondestructive testing.

Dr. Catala-Civera is an IMPI Member. He is currently a Board Member of the Association of Microwave Power in Europe for Research and Education (AMPERE), a European-based organization devoted to the promotion of RF and microwave energy.

Antoni J. Canós was born in Almenara (Castelló de la Plana), Spain, in 1973. He received the Dipl. Eng. and M.S. degrees in electrical engineering from the Universitat Politècnica de València, Valencia, Spain, in 1999 and 2003, respectively, and is currently working toward the Ph.D. degree at the Universitat Politècnica de València, Valencia.

In 2001, he joined the Institute for the Applications of Advanced Information and Communication Technologies (ITACA), Universitat Politècnica de València, as a Research and Development Engineer. Since 2005, he has been an Assistant Professor with the Communications Department, Universitat Politècnica de València. He has coauthored more than 30 papers in referred journals and conference proceedings. He holds four patents. His research interests include numerical analysis and design of waveguide components.

Pedro Plaza-González was born in Elda, Spain, in June 1971. He received the Dipl.Ing. degree in telecommunications engineering from the Universidad Politécnica de Valencia, Valencia, Spain, in 1998, and is currently working toward the Ph.D. degree at the Universidad Politécnica de Valencia.

Since 1999, he has been a Research Assistant with the Microwave Applications Research Division, Institute for the Applications of Advanced Information and Communication Technologies (ITACA), Universidad Politécnica de Valencia. His current research interests are focused on microwave-assisted heating and drying processes and microwave power instrumentation.
José D. Gutiérrez was born in Molinicos (Albacete), Spain, in 1984. He received the Dipl.Eng. in electrical engineering from the Universitat Politècnica de València (UPV), Valencia, Spain, in 2008.

In 2008, he joined the Institute for the Applications of Advanced Information and Communication Technologies (ITACA), UPV, as a Research and Development Engineer and as a Laboratory Technician. His current research interests include microwave measurement techniques and devices for the electromagnetic characterization of materials.

Beatriz García-Baños was born in Madrid, Spain, in 1979. She received the B.S., M.S., and Ph.D. degrees in electrical engineering from the Universitat Politècnica de València, Valencia, Spain, in 2003, 2005 and 2008, respectively.

From 2003 to 2008, she was a Research Assistant with the Universidad Politécnica de Valencia. In 2008, she joined the Microwaves Industrial Applications Division (DIMAS), Institute for the Applications of Advanced Information and Communication Technologies (ITACA), Universidad Politécnica de Valencia, where she is specialized in the development of microwave equipment for dielectric characterization, materials, and process monitoring, and heating processes. She has authored or coauthored more than 30 papers, conference proceedings, and book chapters. Her research interests include microwave sensors and systems for materials characterization.

Felipe L. Peñaranda-Foix (M'10-SM'15) was born in Benicarló, Spain, in 1967. He received the M.S. degree in electrical engineering from the Universidad Politécnica de Madrid, Madrid, Spain, in 1992, and the Ph.D. degree in electrical engineering from the Universidad Politécnica de Valencia (UPV), Valencia, Spain, in 2001.

In 1992, he joined the Departamento de Comunicaciones, UPV, where he is currently a Senior Lecturer. He has coauthored about 40 papers in referred journals and conference proceedings and more than 40 engineering reports for companies. He is a reviewer for several international journals. His current research interests include electromagnetic scattering, microwave circuits and cavities, sensors, and microwave heating applications.

Dr. Peñaranda-Foix is a Member of AMPERE. 